Aus dem Anatomischen Institut der Universität Heidelberg.

Dir. Geheimrat Prof. Dr. Fü rbringer.

\title{
Neue Methoden \\ zur Darstellung des Verlaufs der Blutgefässe bei Amphibienlarven und Hühnerkeimscheiben.
}

Von

Dr. Franz Rost, Assistent.

Hierzu Tafel XXX und XXXI.

Seitdem in der Histologie die chemisch wohlcharakterisierten Anilinfarben in Aufnahme gekommen sind, bemühte man sich, mit ihrer Hilfe einmal die chemische Struktur des Protoplasma zu ergründen, dann suchte man umgekehrt mit bestimmten Farben bestimmte Gewebe elektiv $z \mathbf{u}$ färben und dadurch leichter kenntlich zu machen. Leider sind die Erfolge dieser Bemühungen nicht so glanzende gewesen, als die Erwartungen. Die Zahl der "spezifischen Farbungen ${ }^{\prime}$ ist eine geringe geblieben und die rein empirisch gefundenen entbehren oft der Begründung. Nun sind aber doch immerhin die Gewebe im ausgebildeten Zustand mit unseren bisherigen Methoden meist so gut darstellbar, dass man neue elektive Fărbungen entbehren kann, ja man hat schon manchmal den Eindruck, dass infolge der allzu bunten histologischen Prăparate für den Anfanger das mikroskopische Sehenlernen erschwert ist.

Anders verhalt es sich mit den embryologischen Geweben. Sie sind in jüngeren Stadien chemisch wenig differenziert und heben sich deshalb färberisch nicht immer genügend voneinander ab. Methoden, die hier nachhelfen würden, sind deshalb recht erwünscht, beispielsweise besonders, wenn es sich um die Darstellung und das Studium des Verlaufs der Blutgefasse $\mathrm{b}$ andelt, da junge Blutzellen anderen embryologischen Gewebezellen recht ahnlich sehen können.

Ich ging, um rote Blutzellen von anderen Gewebsarten zu trennen, von dem Gedanken aus, dass es möglich sein müsste, die Kerne geschădigter roter Blutkörperchen innerhalb des lebenden 
Tieres zu färben, was natürlich vollståndig zu ihrer Erkennung genügen würde. Eine solche elektive Farbung der Erythrocyten war mir beim Frosch nach Vergiftung des Tieres mit Hydroxylamin und anderen Blutgiften ausgezeichnet gelungen. ${ }^{1}$ ) Dass auf diese Art die Form und Struktur der Zelle Not leidet, will nicht viel sagen. Wir studieren ja doch eigentlich niemals das Blut morphologisch im Schnitt oder in den Gefässen, sondern stets im Ausstrich. Wir werden solche Vergiftungen deshalb auch nur dort vornehmen, wo es sich um Darstellung der Blut bahn, nicht der Blutzelle $n$ handelt.

Die Versuche wurden an Larven von Rana tempor., Bufo cinereus und Bombinator igneus, auch Triton alpestris in verschiedener Grösse angestellt. Die schönsten Präparate erhielt ich von ersterer Kaulquappenart. Die Versuchszahl ist eine recht beträchtliche. Es wurde eine grosse Zahl von Farben und auch verschiedene Gifte angewendet. Die besten Resultate ergab mir folgende Methode:

Man fügt zu $20 \mathrm{ccm}$ Brunnenwasser $1 / \mathrm{s} \mathrm{ccm} \mathrm{Methylenblau,}$ $1 \%$ Anilinblau Merk und $0,1 \mathrm{ccm}$ einer $1 \%$ igen Lösung von Hydroxylamin hydrochl. ${ }^{2}$ ) Man muss diese leicht saure Mischung mit Sodalösung neutralisieren. Ich neutralisierte die Hydroxylaminlösung von vornherein mit $0,5 \%$ Sodalösung und nahm dann anstatt $0,1 \mathrm{ccm}$ der reinen, $0,2 \mathrm{ccm}$ der neutralisierten Lösung. Methylenblau löst sich meist nicht zu $1 \%$ in Wasser; ich kochte es zur besseren Lösung auf, aber auch dann fiel nach dem Erkalten oft früher oder später etwas von der Farbsubstanz aus. Dann muss man mehr Farbe nehmen; in praxi etwa so viel, dass man in einer Zimmermannsschale noch eben die darin befindlichen Gegenstände, gegen weisses Papier gehalten, erkennt. In diese Mischung tut man die Kaulquappen und beobachtet von Stande zu Stunde ihr Befinden, indem man sie reizt. Reagieren sie noch prompt, braucht man sie kaum erst mikroskopisch zu beobachten. Allmählich - etwa nach 5 Stunden, doch schwankt das sehr werden sie trăge, sind wohl auch schon narkotisiert. Man legt sie dann auf einen Objekttrăger und beobachtet die Blutgefasse

1) Erscheint in Pflügers Archiv d. J. Bd. CXIII, 1.

$\left.{ }^{2}\right)$ B inz: Toxikologisches über das Hydroxylamin. Virch. Arch.,

Lew in: Hydroxylamin. Arch. f. exp. Path. u. Pharm., Bd. 25, S. 306. 1888. 
im Schwanz. In der Regel sind dann schon eine Reihe Kerne von roten Blutkörperchen gefarbt und auch das Protoplasma hat einen grünlichblauen Farbenton angenommen. Spăter löst sich ein Teil des Protoplasmas auf und man sieht freie, gefarbte Kerne in den Blutgefăssen kreisen. In einzelnen derselben kommt es zu Stase.

Die Kernfärbung wird immer intensiver und beim Tode sind ungefåhr alle Kerne der roten Blutkörperchen gefärbt. Wann der Tod eintritt, ist schwer zu sagen. Durchschnittlich hatten die Blutzellen in meinen Versuchen die gewünschte Farbung in 10-12 Stunden erreicht. Man kann auch zuerst einige Stunden das Gift einwirken lassen und dann Farbe binzufügen. Das Resultat ist das gleiche. Ausser Methylenblau erhielt ich gute Erfolge noch mit Thionin und Toluidinblau; eine grosse Zahl anderer Farben war nicht so brauchbar (Neutralrot, Vesuvin und Bismarckbraun, Eosin w. l., Indigkarmin, Nilblausulfat, Orange G., Safranin alle $1 \%$; von den Lösungen $0,1: 20,0$ Wasser; auch andere Mischungsverhältnisse wurden versucht).

Eine elektive Kernfarbung durch spezifische Fixation war damit erreicht und Beobachtung am lebenden Tier gut möglich. Für Dauerpräparate zum Studium der Blutbahnen muss nun das gefärbte Blut möglichst in die peripheren Gefässe getrieben und dann fixiert werden. Nun war es bei dem Gift recht unangenehm, dass die Tiere haufig in diastolischem Herzstillstand starben, und dadurch die schön gefărbten Blutkörperchenkerne zum Schluss in das Herz gepumpt wurden. Anwendung von infus. fol. digit. in wechselnder, oft recht beträchtlicher Menge änderte daran gar nichts. Wohl aber erhielt ich bessere, wenn auch nicht in allen Fällen tadellose Resultate, wenn ich nach genügender Färbung die Tiere lebend in gesăttigte, wăsserige Lösung von Pikrinsảure brachte, die gleichzeitig zur Fixation der Farbung diente. Auf diese komme ich noch zu sprechen. Starb dann das Tier in Systole, so bekam man ein sehr schönes Bild der Gefässverzweigung; denn nur Kerne der roten Blutkörperchen waren gefarbt. Nur an wenigen Stellen kam es auch zur Farbung anderer und zwar der Epithelkerne, die entweder physiologischer Weise abgestorben, aber noch nicht abgestossen waren, oder die durch lange Einwirkung der Farbe, ferner durch Druck (da das Tier durch Hydroxylamin narkotisiert meist am Boden des Gefasses liegt), 
oder durch Säurewirkung (bei nicht genauer Neutralisation) geschädigt worden sind. Alle diese letzterwähnten Fehler kann man durch geeignete, selbstverstandliche Gegenmassregeln aufheben oder einschränken. Die Fixation des Methylenblau bereitet bekanntlich grosse Schwierigkeiten. Bei der vitalen Nervenfärbung gelingt es allerdings mit molybdansaurem Ammon. (5-10\%), wie Dogie l') angibt, sehr haltbare Prăparate herzustellen. Bei der Kernfärbung war ich weniger glücklich. Ich probierte alle möglichen Modifikationen der Betheschen Fixierung ${ }^{2}$ ), auch die

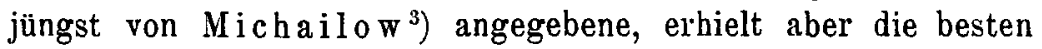
Erfolge mit der Originalmethode, d. h. eine Viertelstunde Aufenthalt der Präparate in gesăttigter, wässeriger Pikrinsăure, darauf obne Abspülen für 24 Stunden in molybdänsaures Ammon. $4 \%$, kurz in dest. Wasser abspülen, übertragen in $96 \%$ Alkohol, absol. Alkohol, Xylol, Balsam. Wenn man die Praparate nach der Ammon.-molybd.Behandlung nur kurz, wie es Bethe angibt, abspült, wird allerlings oft nachtrăgliche Niederschlagsbildung nicht verhindert, wodurch die Präparate rasch verderben. Auch sonst sind sie nicht übermässig haltbar; schon nach 14 Tagen beginnen sie abzublassen und zwar verliert, wie man auch an der Abbildung sieht (Taf. XXX, Fig. 1), zuerst das Protoplasma seinen blauen Ton. Immerhin genügt die Zeit, um genaue Untersuchungen am Präparat vorzunehmen und sich zu überzeugen, dass mit den oben gegebenen Ausnahmen es in der Tat zu einer elektiven Färbung der Kerne der roten Blutkörperchen gekommen ist, herbeigeführt durch ihre Zerstörung mit Hydroxylamin. Ein Nachteil der Methode ist zweifellos die schlechte Fixierbarkeit und, da man diese vielleicht mit der Zeit wird verbessern können, noch mehr der diastolische Herzstillstand. Für Schnitte war die Methode eigentlich aus ersterem Grunde gar nicht recht brauchbar, sondern gab schöne Bilder nur bei Totalpräparaten, wie Schwanzflosse und Extremitat, oder von den Stellen, wo man dünne Stückchen hatte abschneiden oder abziehen können, wie bei Rumpfhaut, Dărmen usw., wobei

1) Methylenblau zur Nervenfärbung. Encycl. d. mikr. Technik, Bd. I, S. 108.1910.

3) Eine neue Methode der Methylenblaufixation. Anat. Anz., Bd. XII, S. 438.1896.

Das Molybdänverfahren etc. Zeitschr.f. wiss. Mikr., Bd. XVI, S.13. 1900.

3) Die Anwendung des Methylenblau in der Neurologie. Ztschr. f. wiss. Mikr., Bd. 27, S. 1. 1910. 
natürlich die topographische Lage zerstört war. Entfernung des Pigments mit den üblichen Methoden (Eau de Javelle) hjelt die Farbung nicht aus. Alles das sind Gründe, die mich einer Verwendung des Verfahrens in praxi vorlaufig etwas skeptisch gegenüberstehen liessen, wăhrend natürlich das prinzipielle Interesse, das diese spezifische Fixation hat, unter diesen Tücken des Objekts nicht leidet.

Ich suchte nun auf andere Weise, namlich durch Erzeugung von Thrombose, die Blutgefăsse der Kaulquappen besser sichtbar zu machen. Nach meinen Versuchen über Kernfärbung am lebenden Frosch eignet sich hierzu gut das Toluilendiamin. ${ }^{1}$ ) Ich stellte mir eine $1 \%$ ige Lösung in Wasser her, die ich genau mit $2 \%$ Essigsăure neutralisierte. Von dieser Lösung tat ich $0,5-1 \mathrm{ccm}$ in 20,0 Wasser und setzte die Larven in diese Mischung. Nach 24 Stunden, oft auch erst spăter, trat dann eine prïchtige Injektion der Blutgefăsse im Schwanze mit einem Brei von Blutkörperchen ein. Da Toluilendiamin besonders bei chronis cher Vergiftung lytisch auf die roten Blutkörperchen wirkt, so ist es empfehlenswerter, die schwächere $(0,5: 20,0)$ Verdünnung anzuwenden, wenn auch dann natürlich die Injektion länger dauert. Ich setzte auch zu diesem Gifte, genau in derselben Weise wie oben, verschiedene Farben und erhielt in der Tat eine schöne diffuse Fărbung des Blutkörperchenbreis. Im allgemeinen empfieblt es sich aber nicht, so vorzugehen. Denn erstens kann es leicht kommen, dass infolge der Thrombose die Farbe nicht gleichmassig verteilt wird, dann werden durch die lange Finwirkung des Toluilendiamin auch andere, besonders Epithelzellen, geschadigt und infolgedessen gefarbt, schliesslich hat man natürlich dieselben Schwierigkeiten der Fixation wie beim Hydroxylamin. Und besonders dieser letztere Grund veranlasste mich, die Farbung nach vorbergegangener Fixation zu versuchen. Fixiert wurden die durch Gift mit Blutkörperchenbrei injizierten Kaulquappen in Formolalkobol. Zur

1) Stadelmann: Das Toluilendiamin und seine Wirkung auf den Tierkörper. Arch. f. exp. Path. u. Pharmakol., Bd. XIV, S. 331 a. 422.

Derselbe : Weitere Beiträge zur Lehre von Ikterus. Ebenda Bd. XVI, S. 118.1883.

Derselbe: Die chronische Vergiftung mit Toluilendiamin. Arch. für exp. Path. u. Pharmakol, Bd. XXIII, S. 427.

Afanassiew: Über Ikterus und Hämoglobinurin, hervorgerufen durch Tolailendiamin etc. Ztschr. f. klin. Med., Bd. XVI, S. 281. 1883. 
Farbung benutzte ich Plasmafarben und Kernfarben. Erstere aus dem Grunde, weil sich bekanntlich alle nekrotischen Gewebe, genau so wie andere gleichmässig strukturierte, organische oder anorganische Gebilde, sehr stark mit Plasmafarben tingieren und sich dadurch scharf von der blasser gefarbten Umgebung abheben. Man kann dieses Verhalten sehr schön an der Abbildung 2 (Taf. XXX) studieren, die nach einer Eosinfarbung gezeichnet worden ist. Bei der zur Anfertigung des Bildes benutzten stärkeren Vergrösserung sieht man, dass sich auch die Kerne der zerstörten Blutkörperchen stark mit Eosin gefärbt haben, ein Verhalten, das andere Kerne, auch wenn sie fixiert sind, nicht zeigen. Dadurch heben sie sich stark ron den übrigen Gewebskernen ab und tragen zur deutlichen Sichtbarmachung der Injektion auch bei schwächster Vergrösserung bei. Zugleich sieht man aber an dem Bilde an einzelnen Stellen Austritt von Erythrocyten aus den Gefüssen, bedingt durch die lange Einwirkung des Toluilendiamin. Es ist ein zweifelloser Nachteil der sonst sehr bequemen Methode. dass man die Kaulquappen so lange in dem Gift belassen muss, bis sich die Blutbahnen injiziert haben. Dieser Fehler fiel fort bei Vergiftung mit Arsenwasserst of $\mathrm{f}$, bei deren Besprechung ich auch die Erfolge mit Kernfarben anführen werde.

Man nimmt in ein Erlenmeyer-Kölbchen 1-11/2 gr acidum arsenicosum auf 30,0 gr Wasser und setzt etwas Lauge hinzu; ${ }^{1}$ ) kocht bei schwacher Flamme so lange, bis sich das Arsen völlig gelöst hat. Den Wasserstoffstrom bereitet man am besten in einem Erlenmeyer-Kölbchen mit doppelt durcbbohrtem Stöpsel aus Zink und Salzsäure. Bevor man die verdünnte Salzsäure auf das Zink laufen lässt, hat man die Arsenlösung in den Kolben gebracht. Dann giesst man durch einen Trichter die Säure zu und leitet den kraftigen, sich rasch entwickelnden Strom von Arsenwasserstoff zunächst in eine Waschflasche mit gewöhnlichem Wasser, dann in das Gefass, in dem sich die Tiere in Wasser befinden. Man setzt die Tiere dem Gas so lange aus, bis alle tot sind, was oft schon in sehr kurzer Zeit $(1 / 4-1 / z$ Stunde) der Fall ist. Bei Larven von Ranu temp. erhält man dann eigentlich in jedem Exemplar eine gleichmåssig schöne Injektion aller sicht-

1) Städelmann: Die Arsenwasserstoffvergiftung. Arch. f. exp. Path. u. Pharmakol., Bd. XVI, S. 221, 1883. 
baren Blutgefässe mit Erythrocytenbrei. Bei Bufo cinereus und Bombinator igneus waren die Erfolge nicht so gleichmässig, doch auch in der Mehrzahl der Tiere vorbanden. Bufo braucht auch langere Zeit bis zum Exitus. Es sind also wohl dieeinzelnen Blutsorten nicht gleichmăssig empfindlich gegen das Gift, was biologisch nicht ohne Interesse ist. Ich erinneredaran, dass esbeim Nenschen ca. 8 Stunden dauert, bis Hamolyse eintritt.')

Die Tiere wurden dann wiederum mit Formolalkohol fixiert und dann gefärbt. Will man Kernfärbung innerhalb des leb enden Tieres haben, so muss man zunachst den Arsenwasserstoffstrom langsamer zuführen, was man mit Klemmen gut regulieren kann. Man nimmt in dem Falle auch vorteilhafter nur 1/2 gr acid. arsenic. als Ausgangsmenge. Fügt man dann, genau wie bei Hydroxylamin, dem Wasser, in dem sich die Tiere befinden, Methylenblau oder eine derartige Farbe hinzu, so kann man in der Tat oft sehr schöne Kernfärbung der Erythrocyten während des Lebens der Larven bekommen, wobei sich zugleich nur sehr wenig Epithelkerne färben, da ja das gewaschene Gas nicht wie Toluilendiamin reizend auf die oberflächlichen Gewebe einwirkt. Doch ist auch in diesem Falle wegen der haufig rasch eintretenden Thrombose das Resultat der Färbung kein selur gleichmässiges und nicht sicheres. Es empfiehlt sich deshalb im allgemeinen die Fixation und nachträgliche Färbung mit Kern- oder Plasmafarben viel mehr. Über letztere ist dem bei Toluilendiamin Gesagten nichts mehr hinzuzufügen, als Beispiel der ersteren füge ich ein Bild ( 3 , Taf. XXX) von Hămalaunfärbung eines Kaulquappenschwanzes von Rana temp. bei. Man sieht, wie die dicht beieinander liegenden Kerne der aufgelösten Erythrocyten sich stark gefărbt baben und dadurch in der Tat den Verlauf der Blutbahnen in einer Weise schön und vollståndig zur Darstellung bringen, wie es nicht besser und vollkommner durch Injektion von Tusche oder Farbe vom Herzen aus geschehen kann. Dabei lässt die Vergiftung mit Arsenwasserstoff an Einfachheit der Ausführung nichts zu wünschen übrig, wăhrend Injektionen von Larven sonst bekanntlich technisch

1) Bei feineren morphologischen Ontersuchungen ist das Blut einander nahestehender Tierklassen durchaus verschieden gebaut. cf. Me ves: Über die Wirkung gefärbter Jodsäure auf die roten Blutkörperchen der Amphibien. Anat. Anz., Bd. 26, S. 102, 1905. 
äusserst schwierig sind. ${ }^{1}$ ) Es kann nun bei Kaulquappen, die ohne jede vorherige Zerstörung der Blutkörperchen getötet, fixiert und gefärbt worden sind, auch öfters beobachtet werden, dass Erythrocyten in Reihenform angeordnet in den peripheren Gefässen liegen bleiben und dadurch $z \mathfrak{u}$ deren besseren Darstellung beitragen. Bei starker Vergrösserung derartiger Stellen können sich dann ähnliche Bilder ergeben, als wie bei den von mir dargestellten. Um den Unterschied der beiden in ihrer Entstehungsursache ja auch gănzlich verschiedenen Bilder deutlich zu zeigen, füge ich noch zwei bei schwacher Vergrösserung gefertigte Figuren bei von einer nicht vorbehandelten (Taf. XXXI, Fig. 1) und einer mit Arsenwasserstoff vorbehandelten (Taf. XXXI, Fig. 2) Larve von Rana temporaria.

Bei der nicht vorbehandelten Kaulquappe sieht man ab und an auf kurze Strecken etwas Blut in den Gefăssen liegen. Dieselben sind sehr eng, der genauere Verlauf ist nicht deutlich $\mathrm{zu}$ sehen. Ich bemerke, dass ich aus einer grösseren Reihe von Kaulquappenschwănzen dabei noch denjenigen ausgewăhlt habe, wo das Blut auf die längsten Strecken hin in den Gefässen lagerte. Ganz anders sieht der Schwanz an dem mit Arsenwasserstoff vergifteten Tiere aus. Während die kleinen Gefasse schon mit Blutkörperchenbrei gefüllt waren, hatte das Herz immer noch mehr zerstörte Erythrocyten nachgepumpt und dadurch die Gefässe auf das ausserste gefüllt. Es sind deshalb bei diesen injizierten Larven die Gefăsse als breite Bănder auch bei schwacher Vergrösserung deutlich $\mathrm{zu}$ sehen und es beschränkt sich diese Thrombose nicht auf umschriebene Stellen, sondern es ist zu einer vollständigen Injektion gekommen und dadurch die Gefasse in ihrem gesamten Verlauf von der Aorta an dargestellt. Dabei werden als Injektionsmaterial arteigene, zerstörte Zellen benutzt und als treibende Kraft zur Verteilung der Masse dient das Herz. Man kann sich eine "physiologischere ${ }^{*}$ Injektion nicht gut vorstellen.

Im allgemeinen eignen sich alle guten Kernfarbstoffe auch gut zur Farbung dieser Objekte. Besonders schöne Resultate

2) Herbert M. Evans. On the Earliest Blood-Vessels in the Anterior Limb Buds of Birds and their Relation to the Primary Subclavian Artery The American Journal of Anatomy. Vol. IX, No. 2. May 1909. S. 283 und 284 . 
erhielt ich noch mit Thionin, ${ }^{1}$ ) Bismarckbraun ${ }^{2}$ ) und Toluidinblau, ${ }^{3}$ ) die ich sehr empfehlen kann. Bezüglich ihrer Anwendung verweise ich besonders auf die angegebene Literatur.

Auch Schnitte fertigte ich von den mit Arsenwasserstoft oder Toluilendiamin vergifteten Tieren an. Bei letzteren war das Einbetten oft unvolistandig (Paraffin), was ich bei ersteren nicht bemerken konnte. Auf den Schnitten zeigten sich dann die kleineren Gefăsse meist mit Blut gefüllt, das zu einem Brei verăndert war. Aorta und Herz enthielten wenig und normale Blutkörperchen. Es kann also in gewissen Fallen die Methode wohl dazu dienen, feinere Verzweigungen der Blutgefăsse sichtbar zu machen, doch glaube ich nicht, dass das ibr Haupt-Anwendungsgebiet sein wird, dazu würde sich die erste Methode der vitalen Färbung, die mit der Fixierbarkeit des Methylenblau steht und fallt, besser eignen. Denn die Verzweigungen der Blutgefassse, die man heutzutage bei Larvenstadien sucht, sind wohl mehr Blutlachen als umschlossene Blutgefasse und lassen sich deshalb durch Arsenwasserstoffvergiftung nicht gănzlich mit Blut füllen, sind vielmehr sicherer auf Serienschnitten zu erkennen. Wichtig ist aber eine Injektion immer, um die Abgangsstellen der Gefässe sich im Totalpräparat gewissermassen plastisch vorzuführen und die durch Serienschnitte gewonnene körperliche Vorstellang des Verlaufes auf diese Art zu kontrollieren. Dazu eignet sich die Methode der künstlichen Thrombosebildung durch Arsenwasserstoff oder Toluilendiamin und nachträgliche Färbung ausgezeichnet. Das illustrieren ja die beigegebenen Abbildungen deutlich, die von Schwänzen der Larven stammen. Will man die Verzweigung der Blutgefasse im Rumpf studieren, muss man bei jeder Art von Injektion die Amphibien depigmentieren. Das ist bisher leider nur recht mangelhaft möglich, wie ich mich

1) Heidenhain: Nene Untersuchungen über das Zentralkörperchen. Arch. f. mikr. Anat., Bd. XLIII, S. 433.1894.

) Weigert: Bismarckbraan als Färbemittel. Arch. f. mikl. Anat., Bd. XV, S. 258.1878.

Born: Die Nasenhöhlen and der Tränennasengang etc. Morph. Jahrb., Bd. V, S. 64. 1879.

s) Mann: Über die Behandlung der Nervenzellen für exp. hist. Unters. Zeitschr. f. wiss. Mikrosk., Bd. XI, S. 479.1894.

H a r r is: The Philadelphia and Journ. 1898. Ref. Zeitschr.f. wiss. Mikrosk., Bd. XVI, S. 60.1899. 
überzeugen konnte. $\left.{ }^{1}\right)$ Ausgezeichnet kann man aber die Verzweigung der Rumpfarterien an $H \ddot{u h n e r k e i m s c h e i b e n}$ beobachten. 60-72 Stunden bebrütete Hühnereier wurden an einer Seite geöffnet, etwas Eiweiss mit der Schere entfernt und das ganze Ej in Lockescher Lösung untergetaucht, die genau auf $37^{\circ}$ gehalten wurde. Es wurde dann der Arsenwasserstoffstrom durch die Lösung geleitet, bis das Herz des Embryo zu schlagen aufhörte, wozu oft mehrere Stunden erforderlich waren. Da man Arsenwasserstoffversuche bei fehlendem Abzug im Freien vornehmen muss und offene Flammen nicht anwenden darf, ist es nicht immer ganz leicht, die erforderliche Temperatur einzuhalten. Ich erreichte es dadurch, dass ich den Behälter mit dem $\mathrm{Fi}$ und der Lockeschen Lösung in ein grösseres Gefäss hing, in dem sich etwas höher temperiertes Wasser befand, in das ich einen jener bekannten Taschenthermophore gebracht hatte. Ia die Versuche nur in die warme Jahreszeit fielen, konnte ich bei haufiger Kontrolle und Nachfüllen von warmem Wasser die Temperatur über viele Stunden konstant halten. Bequemer ist es, das eröffnete Hühnerei in eine Lösung von $2, \tilde{o} \mathrm{ccm}$ T olu il end i a m in $(1 \%): 100,0 \mathrm{ccm}$ L ock e scher Lösung in den Brutschrank zu stellen. Es können die Keimscheiben in dieser Lösung 24 Stunden leben und sind dann ebenfalls durch Thrombose prichtig injiziert. Die Fixation erfolgt in beiden Fallen in Formolalkohol, dann fảrbt man, wie üblich, oder kann auch die Keimscheiben ungefärbt aufheben, nachdem man sie in steigendem Alkohol entwässert und in Xylol aufgehellt hat. Der gelbliche Blutkörperchenbrei hebt sich sehr schön von dem ungefärbten durchsichtigen Körper $a b$ und zeigt den Verlauf der Blutgefasse auf das deutlichste.

\section{Zusammenfassung.}

Zur besseren Sichtbarmachung der Blutgefässe bei Larven von Amphibien kann man entweder die Blutkörperchen durch Hydroxylamin schädigen und dabei ihre Kerne wahrend des Lebens der Tiere fărben. Fixation der Methylenblaufărbung nach Bethe.

1) Versuche über Depigmentation in grösserem Maßstabe und besonders an jungen Exemplaren konnte ich noch nicht anstellen, da die Jahreszeit schon au weit vorgeschritten war, als ich den hier veröffentlichten Teil meiner Versuche abgeschlossen hatte. Ich werde wohl später darauf noch zurückkommen. 
Oder man bewirkt durch Vergiftung mit Toluilendiamin oder Arsenwasserstoff Thrombose und färbt die fixierten Objekte. Die Blutgefăsse heben sich dann ebenso deutlich, wie bei künstlicher Injektion, von der Umgebung ab. Dasselbe gilt für 6u-72 Stunden bebrütete Hühnerkeimscheiben.

\section{Erklärung der Abbildungen auf Taf. XXX und XXXI.}

\section{Tafel XXX.}

Schwänze von Rana tempor.

Fig. 1. Vergiftung mit Hydroxylamin, gleichzeitige Fürbung mit Methylenblau. Fixation in gesättigter wässeriger Pikrinsiiure und molybdänsaurem Ammonium. Vergr.: Zeiss' Obj. DD, Okul. 4, $380: 1$, gez. mit Abbès Prisma.

Fig. 2. Vergiftung mit Toluilendiamin. Fixation in Formolalkohol, Fïrbung in Eosin. Vergr.: Zeiss' Obj. D, Okul. 1, 160:1, Abbès Prisma.

Fig. 3. Vergiftung mit Arsenwasserstoff, Fixation wie Fig. 2, Färbung mit Hämalaun. Vergr.: Zeiss' Obj. A, Okul. 4, 105 : 1, Abbès Prisma.

\section{Tafel XXXI.}

Fig. 1. Schwanz von Rana temp. Unvergiftet fixiert und mit Hämaliun gefärbt. Man sieht nur sehr zart und nicht überall Blut in den Gefässen. Zeiss' Obj. a ${ }^{2}$, Okul. 4, Tubus auf 20 ansgezogen, Vergr.: $60: 1$.

Fig. 2. Schwanz von Rana temp. Vergiftet mit Arsenwasserstoff, Fixation in Formolalkohol, Färbung mit Toluidinblau. Es ist zu vollständiger Injektion der Gefässe mit Blutkörperchenbrei gekommen. Die Gefässe sind als breite Bänder im ganzen Verlauf sichtbar. Zeiss' Obj. a ${ }^{2}$, Okul. 4, Tubus anf 20 ausgezogen, Vergr.: $60: 1$.

Näheres im Text.

Herrn A. Vierling-Heidelberg spreche ich für die künstlerische Ausführung der Bilder meinen besten Dank aus. 


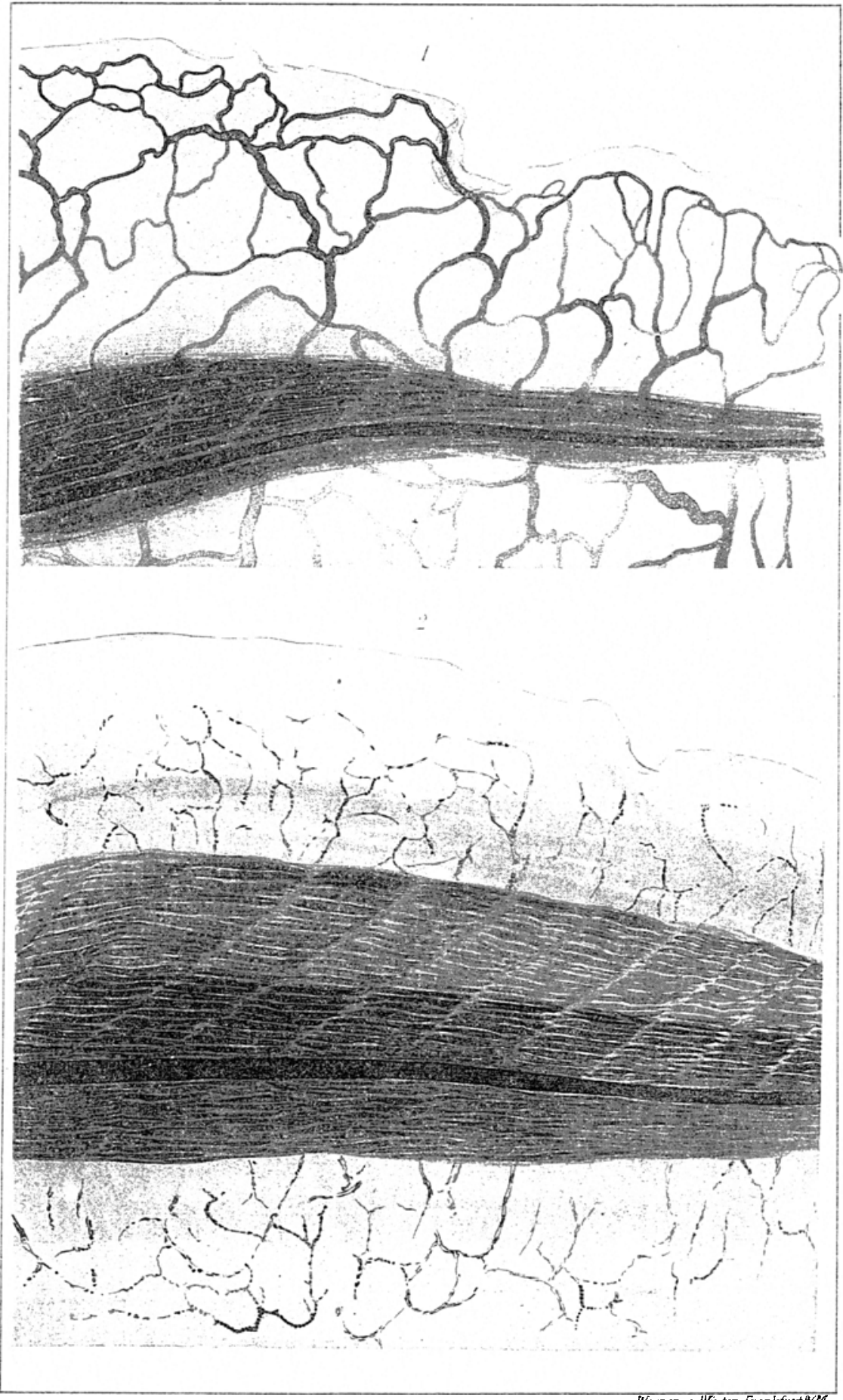

